\title{
A Systematic Model of Big Data Analytics for Clustering Browsing Records into Sessions Based on Web Log Data
}

\author{
Chung Yung ${ }^{*}$, Chia-Ching Chen ${ }^{1}$, Yu-Lan Yuan², Ching Li ${ }^{3}$ \\ ${ }^{1}$ Dept. of Computer Science and Information Engineering, National Dong Hwa University, Taiwan, R.O.C. \\ 2 Dept. of Landscape Architecture, Tunghai University, Taiwan, R.O.C. \\ ${ }^{3}$ Graduate Institute of Sport, Leisure and Hospitality Management, National Taiwan Normal University, \\ R.O.C. \\ * Corresponding author. Tel.: 886-3-8905017; email: yung@mail.ndhu.edu.tw \\ Manuscript submitted December 2, 2018; accepted January 30, 2019. \\ doi: $10.17706 /$ jcp.14.2.125-133
}

\begin{abstract}
This paper presents a systematic model of big data analytics for clustering browsing records into sessions based on the web log data. With the rapid development of the Internet and World Wide Web technologies, the behavior of web users becomes more and more complicated. The analysis on web log data may reveal some hint at the browsing behavior of web users. Since the information of browsing sessions has a great impact on the effectiveness of analysis on web log data, especially in the precision of describing the behavior of web users, this motivates our work in developing a systematic model of clustering browsing sessions. First, we present a five-phase architecture that we develop for big data analytics. We have built a computing environment with the architecture, and we have implemented a few methods of big data analytics with such an architecture. Then, we propose the new systematic model, called EDCP model, of big data analytics for clustering browsing records into sessions based on the web log data. Since the analysis on the web log data with various goals may pose distinct criteria for clustering browsing records into sessions, the design of EDCP model allows simple adaption for the distinct criteria in order to meet the need of various goals. We demonstrate the application of EDCP model with the session criteria given by a research group in the tourism and recreation area. We present the experiments of applying EDCP model on the web log data from the official web site provided by Taiwan Tourism Bureau with a goal of clustering the browsing sessions for the web users of 2018 Taiwan Lantern Festival. As a summary, we have a total of $344,963,578$ browsing records in the web log data, and we find 55,318,326 records among them are related to 2018 Taiwan Lantern Festival. Our systematic model successfully clusters the records into 307,154 browsing sessions, as a result.
\end{abstract}

Key words: Big data analytics, network user behavior, web log mining.

\section{Introduction}

Due to the rapid development in the Internet and World Wide Web (WWW) technologies, web log data is one of the fastest growing user generated contents in cloud computing era [1], and the behavior of web users becomes more and more complicated [2]. The technical papers reporting their efforts in analyzing the behavior of web users by mining the web log data include [2]-[11].

Web mining can be classified into three types: web content mining, web usage mining, and web structure mining [12], and web log mining is usually viewed as part of web usage mining [1], [2]. In general, the 
process of web log mining includes four phases: data processing, session recognition, pattern found, and pattern analysis [2]. Taking a detailed investigation on the phases, we observe that the information of browsing sessions has a great impact on the effectiveness of analysis on web log data, especially in the precision of describing the behavior of web users. This motivates our work in developing a systematic model of big data analytics for clustering browsing records into sessions in order to provide more precise and meaningful browsing sessions for the behavior analysis of web users.

The systematic model of big data analytics that we develop for clustering browsing records into sessions includes four phases: the extracting phase, the defining phase, the clustering phase, and an optional post-processing phase. We called it as EDCP model. Since the analysis on the web log data with various goals may pose distinct criteria for clustering browsing records into sessions, the design of EDCP model allows simple adaption for each distinct criterion in order to meet the need of various goals. We demonstrate the application of EDCP model with the session criteria given by a research group in the tourism and recreation area [12].

We present the experiments of applying EDCP model on the web log data from the official web site provided by Taiwan Tourism Bureau with a goal of clustering the browsing sessions for the web users of 2018 Taiwan Lantern Festival in the spanning time from November 1st, 2017 to March 11 $11^{\text {th }}$ 2018. As a summary, we have a total of 344,963,578 browsing records in the web log, and we find 55,318,326 records among them are related to 2018 Taiwan Lantern Festival. Our systematic model successfully clusters the records into 307,154 browsing sessions, as a result.

The rest of this paper is organized as follows. Section 2 briefly describes the related work to this paper. In section 3, we present a five-phase architecture that we develop for big data analyses with web log data. We propose, in section 4, a new systematic model of big data analytics for clustering browsing records into sessions based on web log data. Section 5 presents our experiments on applying the proposed model to the web log data collected from the official website of Taiwan Tourism Bureau. At last is a brief conclusion.

\section{Related Work}

In literature, there are abundant technical papers reporting their efforts in analyzing the behavior of web users by mining the web log data, including [2]-[11]. Here we briefly describe the work by by Sisoldia et al. [3], by Yu and Korkmaz [4], and by Bayir et al. [5].

In [3], the principle objective of web user sessions clustering by Sisodia et al. is to maximise the intra-group while minimizing the inter-group similarity, when the web users are already clustered into groups in advance. Their method includes four phases: web logs pre-processing, vector space representation of web use sessions, computing page relevance in web user sessions, and clustering augmented web user sessions.

In [4], Yu and Korkmaz investigate web pages structure and find the most evident groups of users and web pages. They note that facing huge amount of web logs, it is not always necessary to group all the users in a web log dataset into different clusters, and sometimes finiding out the major dominant user groups and the corresponding web pages is more important. They propose a new way to search the most evident co-clusters of users and the corresponding web pages in the web log data using the frequent super-sequence mining technique.

In [5], Bayir et al. propose a session model based on a page view, and develop a new session construction method called Smart-SRA, accordingly. Different from the conventional session models, they propose that a session is a set of paths traversed in the web graph that corresponds to a user navigation by following the links on the web pages. 


\section{An Architecture of Big Data Analytics with Web Log Data}

In this section, we present the architecture of big data analytics with web log data. The architecture, first proposed by Yung in 2015 [1], includes five phases as shown in Fig. 1.

1. User Data Generation phase: This phase includes all the activities of generating user data. In addition to web log data, we may also include the generation of other needed data, such as location data of RFID devices and GPS devices.

2. Data Acquisition phase: In this phase, we collect all the data needed for big data analytics. Note that the needed data may be stored in various data repositories of different owners.

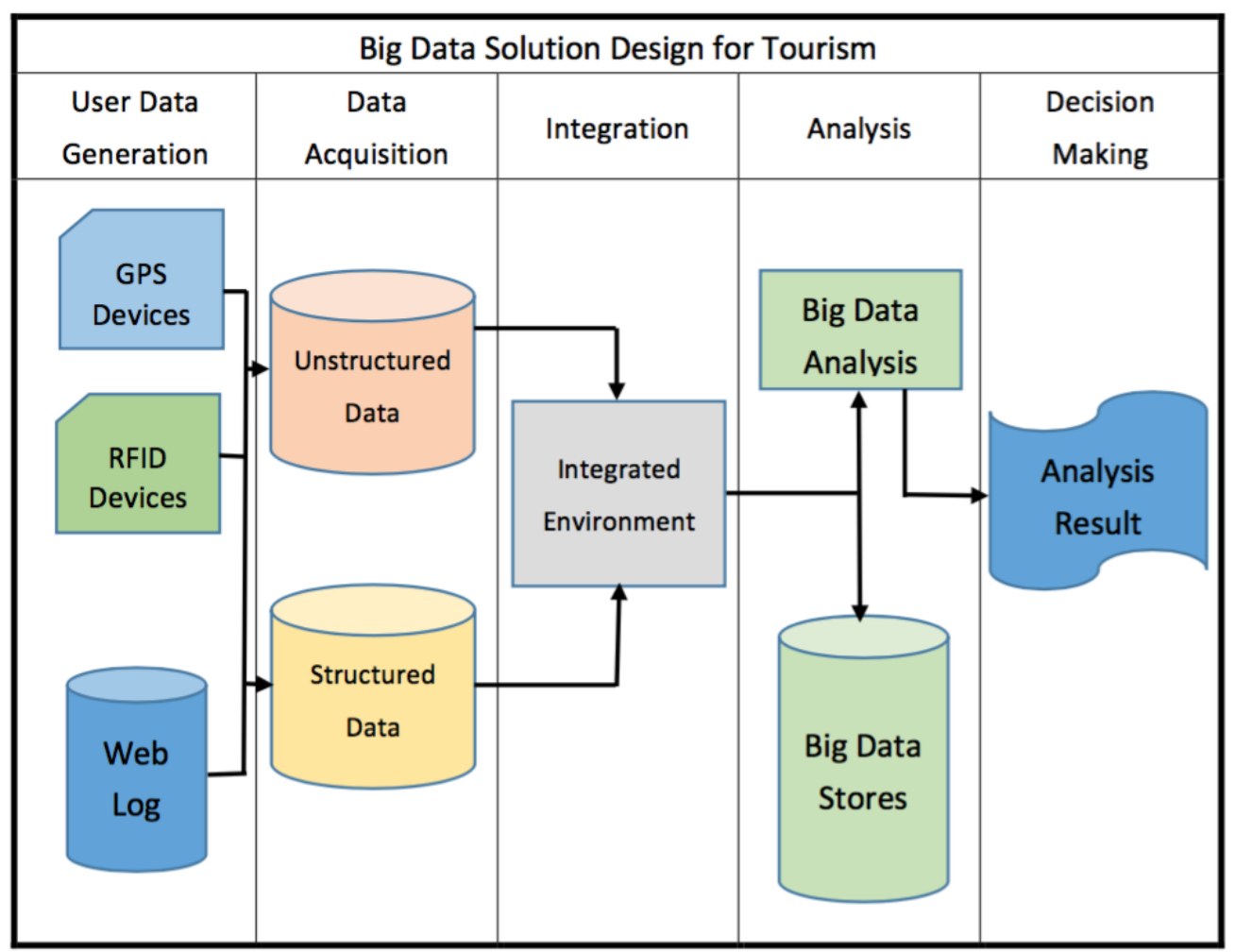

Fig. 1. An architecture of big data analytics with web log data.

3. Integration phase: This phase integrates all the needed data and transforms data when necessary so that the integrated data can be easily processed by the big data analysis algorithms.

4. Analysis phase: One or more analysis algorithms of big data analytics may be chosen for implemention in this phase.

5. Decision Making phase: The final phase is the decision making based on the analysis results of big data analytics. Note that in most of the cases, the ultimate purpose of designing big data analysis is the support of making better decisions.

The five-phase architecture is actually implemented and applied to the experiments presented in this paper.

\section{A New Model of Big Data Analytics for Clustering Browsing Records into Sessions}

In this section, we propose a systematic model of big data analytics, called EDCP model, for clustering browsing records into sessions based on web log data. Please note that EDCP model is applied in the analysis phase of our five-phase architecture of big data analytics with web log data. In other words, our model assumes that all the needed data is collected and integrated into the integrated environment, as 
shown in Fig. 1.

There are four phases in EDCP model, shown in Fig. 2.

1. The extracting phase: The first phase extracts objective records from the massive web log data. Excluding the uninterested records may expedite the procession in the following phases.

2. The defining phase: The second phase defines the criteria for clustering browsing sessions from the web log data. There are two popular ways for defining the session criteria: applying the data mining algorithms to discover the sessions purely from data, or applying the definition of session criteria given by the domain experts. In this paper, our demonstration uses the second one.

3. The clustering phase: The third phase clusters browsing records into sessions according to the criteria defined in the previous phase.

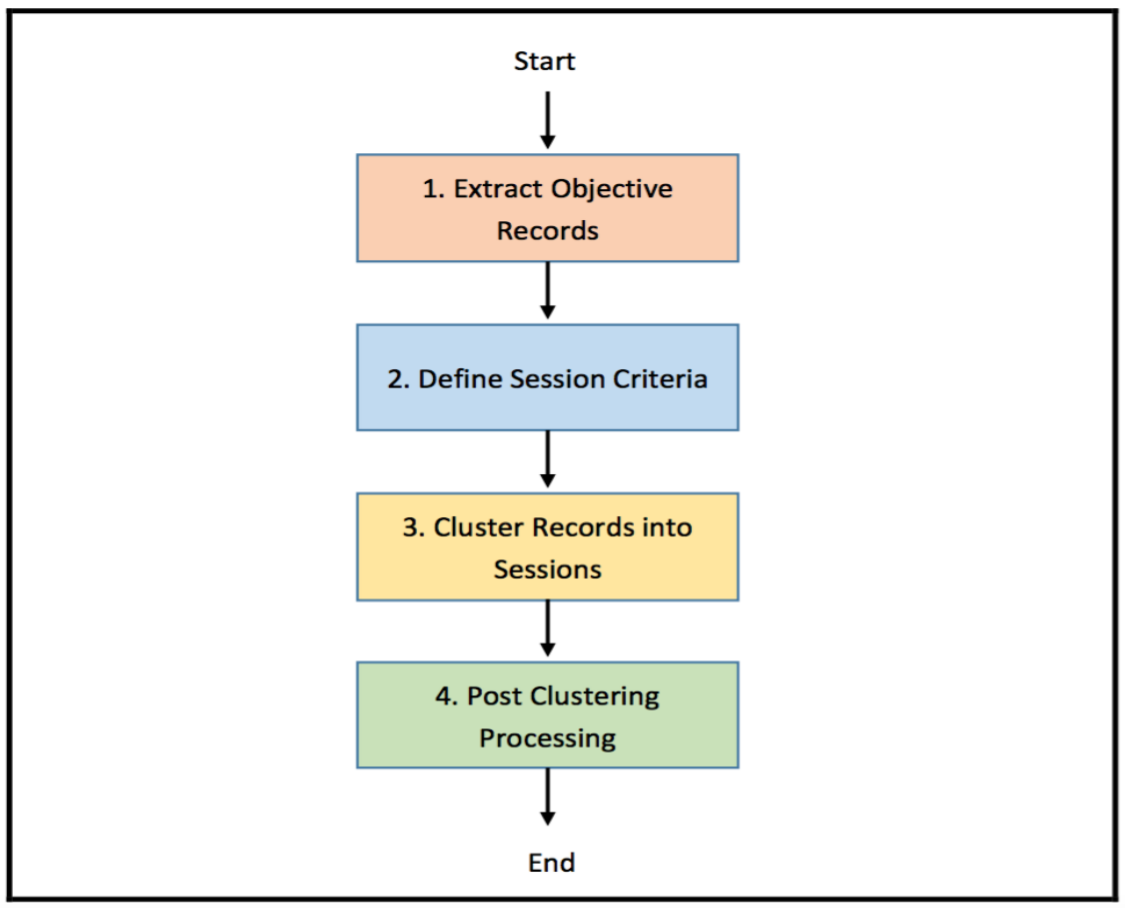

Fig. 2. Phases in the systematic model of big data analytics for clustering browsing records into sessions.

4. The post-processing phase: An optional fourth phase manipulates the procession needed after the sessions are recognized, such as transforming data for the use of follow-up analyses, and extracting the complete data of records if an abbreviated version is used in the previous phases for simplicity.

We call the proposed model as EDCP for the four phases in the model. Note that EDCP model is systematic since we can develop a software program for each phase, and all the programs integrate into a solid software system for clustering browsing records into sessions based on web log data.

\section{Experiments}

In this section, we present our experiments for verifying the validity of EDCP model in clustering browsing records into sessions. We integrate the programs developed in all the phases into an EDCP software system. All programs are developed in $\mathrm{C}++$ programming language. In phase 2, we apply the definition of session criteria given by Li, et al. [12], which may be stated as follows.

If the time difference of two consecutive browsing records of a web user is less than sixty minutes, the 
two records belong to the same browsing session.

In other words, if the time difference of two consecutive browsing records of a web user is greater than or equal to sixty minutes, the two records belong to different browsing sessions.

We experiment on the EDCP software system when applied to the web log data collected between November $1^{\text {st }}$ of 2017 and March 11 $11^{\text {th }}$ of 2018 from the official web site of Taiwan Tourism Bureau [13]. The web log data is integrated into a database system implemented in a MariaDB server [14] before applying the analysis for clustering browsing records into sessions.

\begin{tabular}{|c|c|c|c|c|}
\hline id & I date & time & c_ip & I cs_uri_stem \\
\hline 1 & 2017-11-01 & $00: 08: 45$ & 66.249 .77 .23 & /2018taiwanlantern/0000161.html \\
\hline 2 & $2017-11-01$ & $00: 09: 52$ & 66.249 .77 .25 & /2018taiwanlantern/0000141. html \\
\hline 3 & 2017-11-01 & $00: 10: 27$ & 66.249 .79 .156 & /2018taiwanlantern/EN/News \\
\hline 4 & 2017-11-01 & $00: 12: 41$ & 66.249 .79 .152 & /2018taiwanlantern/TH/O0000004. html \\
\hline 5 & 2017-11-01 & $00: 13: 15$ & 66.249 .79 .156 & /2018taiwanlantern/EN/0000112.html \\
\hline 6 & $2017-11-01$ & $00: 23: 39$ & 126.245 .10 .40 & /2018taiwanlantern \\
\hline 7 & $2017-11-01$ & $00: 23: 39$ & 126.245 .10 .40 & /2018taiwanlantern/ \\
\hline 8 & 2017-11-01 & $00: 23: 39$ & 126.245 .10 .40 & /2018TaiwanLantern/common/css/reset.css \\
\hline 9 & 2017-11-01 & $00: 23: 39$ & 126.245 .10 .40 & /2018TaiwanLantern/common/js/jquery-migrate-3.0.0.min.js \\
\hline 10 & $2017-11-01$ & $00: 23: 39$ & 126.245 .10 .40 & /2018TaiwanLantern/common/css/common.css \\
\hline 11 & $2017-11-01$ & $00: 23: 40$ & 126.245 .10 .40 & /2018TaiwanLantern/common/css/common_rwd.css \\
\hline 12 & $2017-11-01$ & $00: 23: 40$ & 126.245 .10 .40 & /2018TaiwanLantern/common/css/index.çss \\
\hline 13 & $2017-11-01$ & $00: 23: 40$ & 126.245 .10 .40 & /2018TaiwanLantern/common/css/index_rwd.css \\
\hline 14 & $2017-11-01$ & $00: 23: 40$ & 126.245 .10 .40 & /2018TaiwanLantern/common/js/jquery-3.2.1.min.js \\
\hline 15 & 2017-11-01 & $00: 23: 40$ & 126.245 .10 .40 & /2018TaiwanLantern/common/css/1/stylesheet.css \\
\hline 16 & $2017-11-01$ & $00: 23: 40$ & 126.245 .10 .40 & /2018TaiwanLantern/common/css/plugins/slick.css \\
\hline 17 & $2017-11-01$ & $00: 23: 40$ & 126.245 .10 .40 & /2018TaiwanLantern/common/css/plugins/colorbox.css \\
\hline 18 & $2017-11-01$ & $00: 23: 40$ & 126.245 .10 .40 & /2018TaiwanLantern/common/css/plugins/jquery.mmenu. all.css \\
\hline 19 & $2017-11-01$ & $00: 23: 40$ & 126.245 .10 .40 & /2018TaiwanLantern/common/css/plugins/footable.core.css \\
\hline 20 & $2017-11-01$ & $00: 23: 40$ & 126.245 .10 .40 & /2018TaiwanLantern/common/css/plugins/jquery. mCustomscrollbar.css \\
\hline 21 & $2017-11-01$ & $00: 23: 40$ & 126.245 .10 .40 & /2018TaiwanLantern/common/js/common.js \\
\hline 22 & $2017-11-01$ & $00: 23: 40$ & 126.245 .10 .40 & /2018TaiwanLantern/common/js/index.js \\
\hline 23 & $2017-11-01$ & $00: 23: 40$ & 126.245 .10 .40 & /2018TaiwanLantern/common/js/plugins/skrollr.min.js \\
\hline 24 & $2017-11-01$ & $00: 23: 40$ & 126.245 .10 .40 & /2018TaiwanLantern/common/js/zh-tw/google.js \\
\hline 25 & $2017-11-01$ & $00: 23: 40$ & 126.245 .10 .40 & /2018TaiwanLantern/common/js/plugins/imgLiquid-min.js \\
\hline 26 & $2017-11-01$ & $00: 23: 40$ & 126.245 .10 .40 & /2018TaiwanLantern/common/js/plugins/jquery. mmenu.all.min.js \\
\hline 27 & $2017-11-01$ & $00: 23: 40$ & 126.245 .10 .40 & /2018TaiwanLantern/common/js/plugins/slick.min.js \\
\hline 28 & $2017-11-01$ & $00: 23: 40$ & 126.245 .10 .40 & /2018TaiwanLantern/common/js/plugins/jquery.colorbox-min.js \\
\hline 29 & $2017-11-01$ & $00: 23: 40$ & 126.245 .10 .40 & | /2018TaiwanLantern/common/js/plugins/footable.js \\
\hline 30 & $2017-11-01$ & $00: 23: 40$ & 126.245 .10 .40 & | /2018TaiwanLantern/common/js/plugins/jquery.easing.compatibility.js \\
\hline 31 & $2017-11-01$ & $00: 23: 40$ & 126.245 .10 .40 & /2018TaiwanLantern/common/js/plugins/jquery.easing.1.3.js \\
\hline 32 & $2017-11-01$ & $00: 23: 40$ & 126.245 .10 .40 & /2018TaiwanLantern/common/js/plugins/jquery. mCustomscrollbar.concat. min. js \\
\hline 33 & $2017-11-01$ & $00: 23: 40$ & 126.245 .10 .40 & /2018TaiwanLantern/common/js/plugins/jquery-ui.min.js \\
\hline 34 & $2017-11-01$ & $00: 23: 41$ & 126.245 .10 .40 & /2018TaiwanLantern/UserFiles/P1010417.jpg \\
\hline 35 & $2017-11-01$ & $00: 23: 41$ & 126.245 .10 .40 & /2018TaiwanLantern/UserFiles/morning-3.jpg \\
\hline 36 & $2017-11-01$ & $00: 23: 41$ & 126.245 .10 .40 & /2018TaiwanLantern/images/video/video.mp4 \\
\hline 37 & $2017-11-01$ & $00: 23: 41$ & 126.245 .10 .40 & /2018taiwanlantern/json/GetDayNight \\
\hline 38 & $2017-11-01$ & $00: 23: 41$ & 126.245 .10 .40 & /2018TaiwanLantern/UserFiles/20170323_高跟鞋點燈DSC_9493.jpg \\
\hline 39 & $2017-11-01$ & $00: 23: 41$ & 126.245 .10 .40 & /2018TaiwanLantern/UserFiles/morning- $\overline{5} . \mathrm{jpg}$ \\
\hline 40 & $2017-11-01$ & $00: 23: 41$ & 126.245 .10 .40 & /2018TaiwanLantern/UserFiles/morning-8.jpg \\
\hline 41 & $2017-11-01$ & $00: 23: 41$ & 126.245 .10 .40 & /2018TaiwanLantern/UserFiles/night-7.jpg \\
\hline 42 & $2017-11-01$ & $00: 23: 41$ & 126.245 .10 .40 & /2018taiwanlantern/images/weather/02.svg \\
\hline 43 & $2017-11-01$ & $00: 23: 41$ & 126.245 .10 .40 & /2018TaiwanLantern/UserFiles/night-3.jpg \\
\hline 44 & $2017-11-01$ & $00: 23: 41$ & 126.245 .10 .40 & /2018TaiwanLantern/UserFiles/night-4.jpg \\
\hline 45 & $2017-11-01$ & $00: 23: 41$ & 126.245 .10 .40 & /2018taiwanlantern/images/chiayi_map.png \\
\hline
\end{tabular}

Fig. 3. Extracted objective browsing records from web log data.

We include a few figures to show the snapshots of executing the EDCP software system. Fig. 3 presents the extracted objective browsing records after the extracting phase. Fig. 4 shows the two phases in clustering browsing records into sessions: Fig. 4(a) shows the numbering of the visited web pages as the first part of the clustering phase, and Fig. 4(b) shows the messages in the process of clustering browsing records into sessions as the second part of the clustering phase. And, Fig. 5 lists the clustered sessions.

We summarize the experiement with the numbers collected, shown in Table 1. The original web log data has a total of $344,963,578$ browsing records, from which the EDCP software system extracts 55,318,326 objective browsing records that are relevant to 2018 Taiwan Lantern Festival. According to the definition of session criteria given by Li et al., the EDCP software system clusters the objective browsing records into 307,154 browsing sessions. Hence, in average, there are 180.10 browsing records and 5.64 web pages per browsing session. The overall process takes about 4 hours and 45 minutes for the EDCP software system to complete the clustering of browsing records into sessions based on web log data, executed on a desktop system with an Intel i7 CPU and 8 gigabytes RAM . 


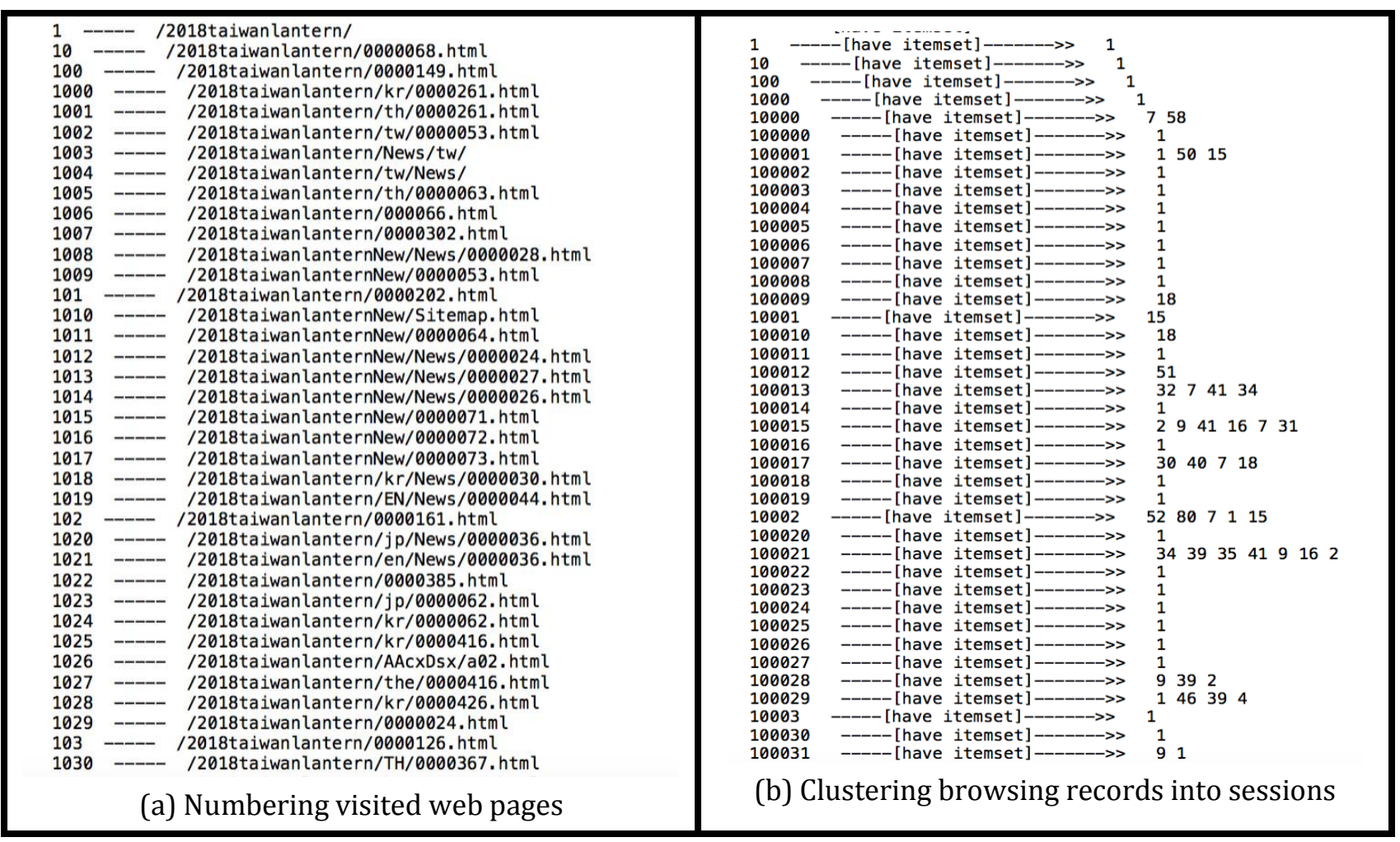

Fig. 4. Two phases in clustering browsing records into sessions.

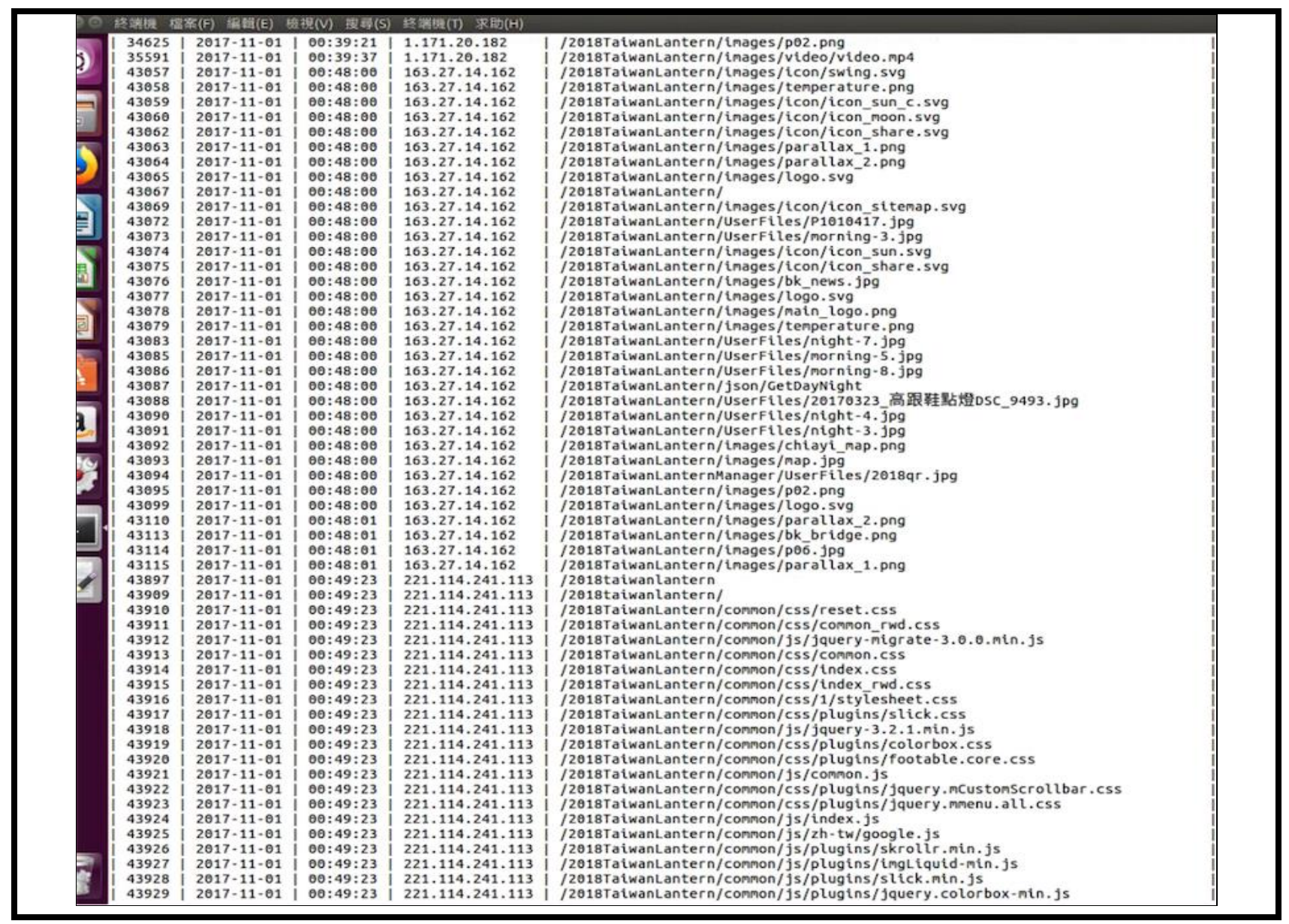

Fig. 5. Clustered browsing sessions. 
Table 1. The Summary of the EDCP Experiment

\begin{tabular}{lrc}
\hline \hline Item & \multicolumn{1}{c}{ Amount } & Unit \\
\hline Original web log data & $344,963,578$ & Records \\
\hline Objective web log data & $55,318,326$ & Records \\
\hline Cluster sessions & 307,154 & Sessions \\
\hline Average records per session & 180.10 & Records \\
\hline Average web pages per session & 5.64 & Pages \\
\hline \hline
\end{tabular}

\section{Conclusion}

In this paper, we present a systematic model of big data analytics for clustering browsing records into sessions based on the web log data. We, first, present a five-phase architecture that we develop for big data analytics. Then, we propose a systematic model of big data analytics for clustering browsing records into sessions, called EDCP model. EDCP model consists of four phases: the extracting phase, the defining phase, the clustering phase, and an optional post-processing phase. We demonstrate the application of EDCP model with the session criteria given by a research group in the tourism and recreation area.

For verifying the validity, we experiment on applying the EDCP model to the web log data from the official web site provided by Taiwan Tourism Bureau with a goal of clustering the browsing sessions for the web users of 2018 Taiwan Lantern Festival in the spanning time from November 1st 2017 to March 11 2018. As a summary, we have a total of $344,963,578$ browsing records in the web log data, and we find 55,318,326 records among them are related to 2018 Taiwan Lantern Festival. Our systematic model successfully clusters the records into 307,154 browsing sessions, as a result.

\section{Acknowledgment}

The authors appreciate Taiwan Tourism Bureau for their kindness in offering the original web log data of their official web site. The work of the third author is partially supported by the Mission of Science and Technology of Taiwan, R.O.C., under grant number MOST 106-2410-H-029-070.

\section{References}

[1] Yung, C. (2015). Mining massive web log data of an official tourism web site as a step towards big data analysis in tourism. Proceedings of the 5th ASE International Conference on Big Data (BigData 2015), (Article F3-03). Kaohsiung, Taiwan, R.O.C.

[2] Luo, X., Wang, J., Shen, Q., Wang, J., \& Qi, Q. (2017). User behavior analysis based on user interest by web log mining. Proceedings of the 27th International Telecommunication Networks and Applications Conference (ITNAC) (pp. 1-5). Melbourne, VIC, Australia.

[3] Sisodia, D. S., Verma, S., \& Vyas, O. P. (2017). Augmented intuitive dissimilarity metric for clustering of web user sessions. Journal of Information Science (JIS), 43(4), 480-491.

[4] Yu, X., \& Korkmaz, T. (2014). Finding the most evident co-clusters on web log dataset using frequent super-sequence mining. Proceedings of the IEEE 15th International Conference on Information Reuse and Integration (IRI 2014) (pp. 529-536). San Francisco, CA, USA.

[5] Bayur, M. A., Toroslu, I, H., Demirbas, M., \& Cosar, A. (2012). Discovering better navigation sequences for the session construction problem. Journal of Data \& Knowledge Engineering (DKE), 73, 58-72.

[6] Hernandez, P., Garrigos, I., \& Mazon, J. N. (2010). Modeling web logs to enhance the analysis of web usage data. Proceedings of the 2010 Workshop on Database and Expert Systems Applications (DEXA) (pp. 291-301). Bilbao, Spain.

[7] Sen, R., \& Hansen, M. (2003). Predicting web users' next access based on log data. Journal of Computational and Graphical Statistics, 12(1), 143-155. 
[8] Agosti, M., Crivellari, F., \& Di Nunzio, G. M. (2012). Web log analysis: A review of a decade of studies about information acquisition, inspection and interpretation of user interaction. Data Mining and Knowledge Discovery, 24(3), 663-696.

[9] Duan, J., \& Liu, S. (2012). Research on web log mining analysis. Proceedings of the 2012 International Symposium on Instrumentation and Measurement, Sensor Network and Automation (IMSNA 2012), 2 (pp. 1981-1985). Sanya, China.

[10] Du, H. S., \& Wagner, C. (2006). Weblog success: Exploring the role of technology. International Journal of Human-Computer Studies, 54(9), 789-798.

[11] Wang, C. H., Tsai, C. T., Fan, C. C., \& Yuan, S. M. (2014). A hadoop based weblog analysis system. Proceedings of the 7th International Conference on Ubi-Media Computing and Workshops (U-MEDIA 2014) (pp. 72-77). Ulaanbaatar, Mongolia.

[12] Li, C., Yuan, Y. L., \& Yung, C. (2017). Definition of browsing sessions for www users of tourism websites. Personal Communication.

[13] Tourism bureau. (2018). Massive Web Log Data of Official Tourism Bureau Website. Taiwan, R.O.C.

[14] MariaDB foundation. (2018). MariaDB Servers. Retrieved May, 2018, from https://mariadb.org/

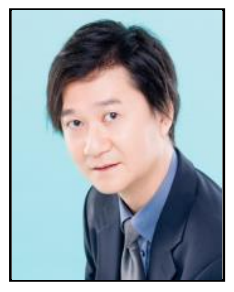

Chung Yung received the Ph.D degree in computer science from New York University (USA) in 1999 and the BS.c degree in computer science and information engineering from National Chiao Tung University (Taiwan) in 1988.

He has been with the Department of Computer Science and Information Engineering of National Dong Hwa University (Taiwan) since 2000. He was a part-time senior consultant and project manager within the intelligent digital content industry between 2003 and 2007.

He is currently leading laboratory of compiler technology and application in National Dong Hwa University. His research interests include systematic big data analytics, semantic methods of program analysis, optimizations for cloud software systems, compiler supported software engineering, and programming languages.

Dr. Yung has been a committee member of CTHPC (Taiwan) since 1999. He received the best presentation award in the 2015 international conference on information technology (Singapore), and the excellent paper award in the 2017 international conference on innovative engineering technologies (Japan). He has been awarded a couple of times for his excellence in research by National Dong Hwa University.

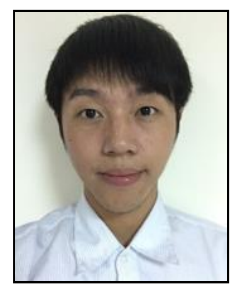

Chia-Ching Chen received a B.A. degree in sociology from National Dong Hwa University (Taiwan) in 2016.

He is currently a graduate student in Department of Computer Science and Information Engineering, National Dong Hwa University. He also works as a part-time software engineer in the laboratory of compiler technology and application.

His current research interests include systematic big data analytics, web content and usage mining, and data mining.

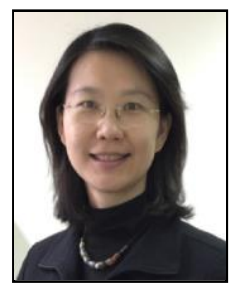

Yulan (Yoyo) Yuan is an associate professor in the Department of Landscape Architecure at Tunghai University (Taiwan). She received the Ph.D degree in Department of Park, Recreation and Tourism from University of Illinois at Urbana-Champaign in 2001, and Big Data Specialist Certificate from Massachusetts Institute of Technology (U.S.A.) in 2015.

Her research interests include spatial data analysis for landscape planning and destination management. Her works have published in top ranked journals in the tourism 
fields, including Annals of Tourism Research, Journal of Travel Research, and Tourism Management. Currently, her team is working on examining the data quality from various sources in assisting tourism management for protected areas.

Dr. Yuan is also the board member of Asia Pacific Tourism Association. She received the ICSLHM best paper award in 2018, the APTA best paper award in 2015, and the best destination management organization case study award from IFITT in 2014.

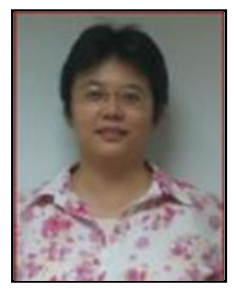

Ching $\mathbf{L i}$ is a professor in Graduate Institute of Sport, Leisure and Hospitality Management, National Taiwan Normal University (Taiwan). She received her Ph.D degree in environmental science from State University of New York (U.S.A.). Her research expertise focuses on recreational resource management, environmental planning and assessment, and leisure behavior. 